\title{
Generalized-stacking-fault energy surface and dislocation properties of aluminum
}

\author{
Gang Lu and Nicholas Kioussis \\ Department of Physics, California State University Northridge, Northridge, California 91330-8268 \\ Vasily V. Bulatov \\ Lawrence Livermore National Laboratory, Livermore, California 94550 \\ Efthimios Kaxiras \\ Department of Physics, Harvard University, Cambridge, Massachusetts 02138
}

(Received 26 January 2000)

\begin{abstract}
We have employed the semidiscrete variational generalized Peierls-Nabarro model to study the dislocation properties of aluminum. The generalized-stacking-fault (GSF) energy surface entering the model is calculated by using first-principles density functional theory (DFT) and the embedded-atom method (EAM). Various core properties, including the core width, dissociation behavior, energetics, and Peierls stress for different dislocations have been investigated. The correlation between the core energetics and the Peierls stress with the dislocation character has been explored. Our results reveal a simple relationship between the Peierls stress and the ratio between the core width and the atomic spacing. The dependence of the core properties on the two methods for calculating the GSF energy (DFT vs EAM) has been examined. Although the EAM gives the general trend for various dislocation properties, it fails to predict the correct finer core structure, which in turn can affect the Peierls stress significantly (about one order of magnitude).
\end{abstract}

\section{INTRODUCTION}

Dislocations which are one-dimensional topological defects, are central to the understanding of mechanical properties of crystalline solids. The creation and motion of dislocations mediate the plastic response of a crystal to external stress. While the continuum elasticity theory describes well the long-range elastic strain of a dislocation for length scales beyond a few lattice spacings, it breaks down near the singularity in the region surrounding the dislocation center, known as the dislocation core. The discrete nature of the real crystalline lattice avoids the conceptual difficulty posed by the continuum singularity and recovers the structural differentiation smoothed out by the continuum elasticity. There has been a great deal of interest in describing accurately the dislocation core structure on an atomic scale because of its important role in many phenomena of crystal plasticity. ${ }^{1,2}$ The core properties control, for instance, the mobility of dislocations, which accounts for the intrinsic ductility or brittleness of solids. The core is also important for the interaction of dislocations at close distances, which are relevant to plastic deformation. For example, by integrating the local rules derived from atomistic simulations of core interactions into dislocation-dynamics simulations, a connection between micro-to-meso scales can be established to study dislocation reactions and crystal plasticity. ${ }^{3}$

Two types of theoretical approaches have been employed to study the core properties of dislocations. The first type, is based on direct atomistic simulations employing either empirical potentials or first-principles calculations. Empirical interatomic potentials involve the fitting of parameters to a predetermined database and hence may not be reliable in describing the core properties, where severe distortions like bond breaking, bond formation and switching necessitate a quantum-mechanical description of the electronic degrees of freedom. On the other hand, first-principles electronic structure calculations, though considerably more accurate, are computationally expensive for studies of dislocation properties. The second type, is based on the framework of the Peierls-Nabarro (PN) model which seems to be a plausible alternative to direct atomistic simulations. In fact, there has been a resurgence of interest in the simple and tractable PN model for the study of dislocation core structure and mobility. ${ }^{4-8}$

Peierls first proposed ${ }^{9}$ the remarkable hybrid model in which some of the details of the discrete dislocation core were incorporated into an essentially continuum framework. Nabarro ${ }^{10}$ and Eshelby ${ }^{11}$ further developed Peierls' model and gave a meaningful estimate of the lattice friction to dislocation motion. Later attempts to generalize the original treatment of Peierls and Nabarro assumed a more general core configuration from which they derived the interactions between the glide planes which satisfy the Peierls integral equation. The essence of these models was captured in a more comprehensive approach by Vitek, ${ }^{12,13}$ who introduced the concept of the generalized-stacking fault: Consider a perfect crystal cut across a single plane into two parts which are then subjected to a relative displacement through an arbitrary vector $\mathbf{f}$ and rejoined. The reconnected lattice has a surplus energy per unit area $\gamma(\mathbf{f})$. As the vector $\mathbf{f}$ is varied to span a unit cell of the interface, $\gamma(\mathbf{f})$ generates the generalizedstacking-fault (GSF) energy surface. The procedure can be repeated for various crystal planes. The significance of the GSF surface (or $\gamma$ surface) is that for a fault vector $\mathbf{f}$ there is an interfacial restoring stress

$$
\mathbf{F}_{b}(\mathbf{f})=-\nabla(\gamma(\mathbf{f}))
$$


which has the same formal interpretation as the restoring stress in the PN model. The PN model has now come to represent a combination of the original continuum model and the GSF interplanar potential, and its accuracy can be affected by either component. At present, the GSF energies can be calculated using empirical interatomic potentials (such as the embedded-atom method, EAM) or electronic structure methods. While extremely useful as a conceptual framework, the PN model becomes increasingly inaccurate for dislocations with narrow cores, which is typically the case in covalently bonded solids. ${ }^{4,14}$ The origin of this inaccuracy remains controversial, and it has not been unequivocally established whether or not the Peierls framework can be extended to capture such situations. Exploring the limits and extending the range of applicability of the classic PN model remains a worthwhile endeavor. Recently, there have been several attempts to generalize the original treatment of the PN model. A semidiscrete variational generalized PN model has been proposed by Bulatov and Kaxiras, ${ }^{15}$ which has been successfully implemented to the study of dislocation mobility in silicon. Using the GSF energy surface determined from an empirical interatomic potential, the model predicts that the Peierls stress for the glide screw dislocation in $\mathrm{Si}$ is 0.065 $\mathrm{eV} / \AA^{3}$, more than two orders of magnitude lower than the value $9.0 \mathrm{eV} / \AA^{3}$ obtained from the classic PN model. Direct atomistic calculations using the same interatomic potential gives $0.021 \mathrm{eV} / \AA^{3}$. Furthermore, the model gives satisfactory results for other core properties when compared to direct atomistic simulations for various dislocations in Si. Considering the effect of the anisotropy of the elastic energy, Schoeck ${ }^{7}$ adopted a variational procedure for the total energy by assuming a $\tan ^{-1}$-type solution for the displacement, which may not be the case for the general interplanar potential. Von Sydow et al. introduced an interesting treatment of the Peierls integrodifferential equation by extrapolating the atomic positions away from the glide plane. ${ }^{8}$ This approach was shown to yield results in better agreement with atomistic simulations employing the same interatomic potential.

The purpose of this paper is to apply the semidiscrete PN model to aluminum which is a prototypical ductile metal with much lower Peierls energy and stress than silicon. On the other hand, just like silicon, aluminum is known to have a narrow core due to its large stacking-fault energy. The successful application of the model to aluminum will further prove its validity and versatility in predicting dislocation core properties for different materials. In particular, aluminum is exceptional among the fcc materials in that there have been no experimental observations for a full dislocation dissociation into partials. ${ }^{17}$ The ability to predict the absence of dissociation in aluminum constitutes another test for the reliability of the model. In order to test the accuracy of the model, we have calculated the Peierls stress using both the semidiscrete generalized PN model and direct atomistic simulations based on the same EAM potential. We have carried out systematic calculations of the core properties and the mobility of relevant dislocations in $\mathrm{Al}$, and we have examined the relationship between the core properties (energetics, core width, and Peierls stress) and the dislocation character, namely the angle between the dislocation line and its Burgers vector. In order to explore the dependence of the dislocation properties on the method employed to calculate the GSF en- ergy, we have performed calculations using both firstprinciples electronic structure methods and the empirical EAM potential.

The remainder of this paper is organized as follows: Sec. II describes the computational techniques used in our firstprinciples calculations for the GSF energy surface. Section III contains a brief review of the model on which this study is based. In Sec. IV, we present the results of the GSF energy surfaces for the (111) plane of aluminum using both firstprinciples and empirical potential calculations. In Sec. V we compare the dislocation properties using the GSF surface evaluated from the two methods. The correlation between the dislocation properties and the dislocation character is presented in Sec. VI, along with some general conclusions on the applicability of our approach.

\section{COMPUTATIONAL METHODS}

The GSF energy surface is calculated within the framework of density-functional theory ${ }^{18}$ (DFT) in the localdensity approximation ${ }^{19}$ to the exchange-correlation functional, using the expression proposed by Perdew and Zunger. ${ }^{20}$ A kinetic energy cutoff of 12 Ry for the planewave basis is used and the atomic structures are considered fully relaxed when the Hellmann-Feynman forces on each atom are smaller than $0.001 \mathrm{Ry} / \mathrm{au}$. The calculated equilibrium lattice constant $\left(a_{0}\right)$ and bulk modulus are $3.94 \AA$ and $82.52 \mathrm{GPa}$, in good agreement with the corresponding experimental room-temperature values of $4.05 \AA$ and 76.93 $\mathrm{GPa}$, respectively.

In addition to the lattice constant and bulk modulus, we have calculated the value of the intrinsic stacking fault energy and the unstable stacking fault energy. To simulate the block shearing process we employed a supercell consisting of six and nine atomic layers in the $\langle 111\rangle$ direction. The intrinsic stacking-fault configuration corresponds to a slip of $a_{0} / \sqrt{6}$ in the $\langle 112\rangle$ direction, resulting in the stacking $A B C \mid B C A B C$. The unstable stacking-fault energy corresponds to the lowest energy barrier that needs to be crossed for the slip from the ideal configuration to the intrinsic stacking fault in the $\langle 112\rangle$ direction. The calculations are performed at the theoretically determined in-plane lattice constant. For the reciprocal-space integration we have used a $k$-point grid consisting of $(16,16,4)$ divisions along the reciprocal-lattice directions according to the Monkhorst-Pack scheme. ${ }^{21}$ This corresponds to $514 k$ points in the entire Brillouin zone. Convergence tests were performed both for the number of divisions along each reciprocal space direction as well as the number of plane waves. Furthermore, the results for the six-layer and nine-layer supercells are reasonably close, indicating adequate convergence with respect to the supercell size. Both atomic relaxations and volume relaxations were carried out to obtain accurate GSF energies. The value of the intrinsic stacking-fault energy we obtained is $0.164 \mathrm{~J} / \mathrm{m}^{2}$, in excellent agreement with the result of 0.165 $\mathrm{J} / \mathrm{m}^{2}$ of Sun and Kaxiras ${ }^{22}$ and that of $0.161 \mathrm{~J} / \mathrm{m}^{2}$ of Wright, Daw, and Fong. ${ }^{23}$ Experimental measurements range from a low value of $0.110 \mathrm{~J} / \mathrm{m}^{2}$ to a high value of $0.280 \mathrm{~J} / \mathrm{m}^{2} .16$ Finally, the value of the relaxed unstable stacking-fault energy is $0.224 \mathrm{~J} / \mathrm{m}^{2}$, in agreement with previous theoretical calculations. ${ }^{5,6}$ 


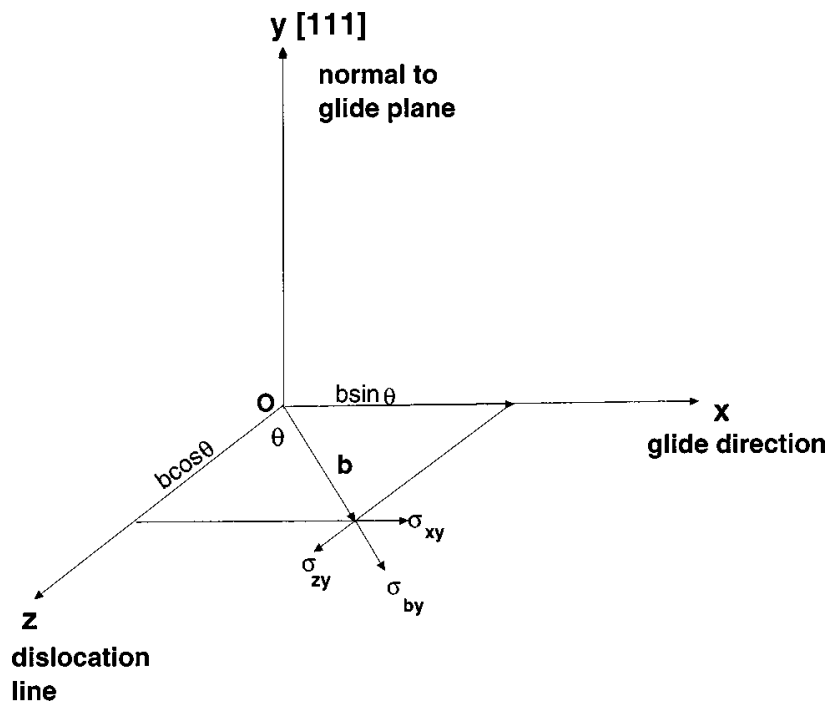

FIG. 1. Cartesian set of coordinates showing the directions relevant for dislocations in $\mathrm{Al}$.

For the EAM calculations of the GSF energy surface, we have used the Ercolessi-Adams potential ${ }^{24}$ for Al. The supercell contains six (111) layers with a total of 216 atoms. Three-dimensional periodic boundary conditions were imposed and both volume and atomic relaxations were included.

\section{SEMIDISCRETE VARIATIONAL PEIERLS-NABARRO MODEL}

To facilitate the presentation, we adopt the following conventions: In Fig. 1, the $X Z$, plane is the (111) glide plane, the $Z$ axis is in the direction of the dislocation line, and the $X$ axis is in the glide direction. The $Y$ axis in normal to the glide plane. For planar dislocations, the displacements along the $Y$ direction are small. The Burgers vector $\mathbf{b}$ lies on the glide plane making an angle $\theta$ with the $Z$ axis. The Burgers vector is along the $X$ axis $\left(\theta=90^{\circ}\right)$ for an edge dislocation and along the $Z$ axis $\left(\theta=0^{\circ}\right)$ for a screw dislocation. The Burgers vector of a mixed dislocation has both an edge component, $b \sin \theta$, and a screw component, $b \cos \theta$. In general, the atomic displacements have components in all three directions rather than only along the direction of the Burgers vector, because the path along the Burgers vector may have to surmount a higher interplanar energy barrier in the GSF surface. In other words, the GSF energy is reduced when the dislocation acquires additional displacement components in other directions.

In the classic PN formalism, the dislocation misfit is assumed to be confined on a single plane, the glide plane, separating two semi-infinite linear elastic continua. Between these two elastic half-spaces one places a dislocation, conveniently represented as a continuous distribution of infinitesimal dislocations with density $\rho(x)$. ${ }^{11}$ Here $x$ is the coordinate of the atomic row, which is always parallel to the dislocation line. A discrete lattice of arbitrary structure, deformed by the dislocation's displacement field, is superimposed on the elastic half-crystals. At a given point along the interface the resultant misfit, due to all the infinitesimal dislocations, is then balanced against the lattice restoring stress across the glide plane, $F_{b}(f(x))$. This results in the PN integrodifferential equation

$$
K \int_{-\infty}^{+\infty} \frac{1}{x-x^{\prime}} \frac{d f\left(x^{\prime}\right)}{d x^{\prime}} d x^{\prime}=F_{b}(f(x)),
$$

where $f(x)$ is the disregistry vector of the atomic row at point $x$ related to the dislocation density by $\rho(x)$ $=d f(x) / d x$, and $K$ is a constant depending on the elastic properties and the dislocation character. For an isotropic solid,

$$
K=\frac{\mu}{2 \pi}\left(\frac{\sin ^{2} \theta}{1-\nu}+\cos ^{2} \theta\right)
$$

where $\mu$ and $\nu$ are the shear modulus and Poisson's ratio, respectively, and $\theta$ is the angle between the dislocation line and the Burgers vector. The prelogarithmic elastic energy factor is $K_{s}=\mu / 2 \pi$ and $K_{e}=\mu /[2 \pi(1-\nu)]$ for a screw and an edge dislocation, respectively. In the following calculations, $\mu=28.8 \mathrm{GPa}=0.1797 \mathrm{eV} / \AA^{3}, \nu=0.344$, and $b=|\mathbf{b}|$ $=2.85 \AA$ are used. The dislocation density $\rho(x)$ satisfies the normalization condition

$$
\int_{-\infty}^{+\infty} \rho\left(x^{\prime}\right) d x^{\prime}=\int_{-\infty}^{+\infty} \frac{d f\left(x^{\prime}\right)}{d x^{\prime}} d x^{\prime}=b .
$$

If a simple sinusoidal form is assumed for $F_{b}(f(x))$, as in the original PN model, the disregistry vector is then given by the well-known analytical solution,

$$
f(x)=\frac{b}{\pi} \tan ^{-1} \frac{x}{\zeta}+\frac{b}{2},
$$

where

$$
\zeta=\frac{K b}{2 F_{\max }}
$$

is the half width of the dislocation core and $F_{\max }$ is the maximum restoring stress.

As pointed out recently by Bulatov and Kaxiras, ${ }^{15}$ the classic PN continuum model has the following flaws: (1) While the elastic energy between the infinitesimal dislocations is evaluated from a continuous integration, the misfit energy across the glide plane is sampled discretely, in order to incorporate the discrete nature of lattice. Thus, the two energy contributions are not treated on an equal footing and the total energy is not variational. (2) The classic PN model neglects the important degrees of freedom which participate actively in the translation of a dislocation over the Peierls barrier, and (3) The elastic strain energy of a dislocation calculated within the PN model can be unrealistically high, especially for solids with a narrow core.

In order to resolve these problems, the semidiscrete variational generalized Peierls model has been developed recently. ${ }^{15}$ Within this approach, the equilibrium structure of a dislocation is obtained by minimizing the dislocation energy functional

$$
U_{d i s l}=U_{\text {elastic }}+U_{\text {misfit }}+U_{\text {stress }}+K b^{2} \ln L,
$$

where 


$$
U_{\text {elastic }}=\sum_{i, j} \frac{1}{2} \chi_{i j}\left[K_{e}\left(\rho_{i}^{(1)} \rho_{j}^{(1)}+\rho_{i}^{(2)} \rho_{j}^{(2)}\right)+K_{s} \rho_{i}^{(3)} \rho_{j}^{(3)}\right],
$$

$$
\begin{gathered}
U_{\text {misfit }}=\sum_{i} \Delta x \gamma_{3}\left(\mathbf{f}_{i}\right), \\
U_{\text {stress }}=-\sum_{i, l} \frac{x_{i}^{2}-x_{i-1}^{2}}{2}\left(\rho_{i}^{(l)} \tau_{i}^{(l)}\right),
\end{gathered}
$$

with respect to the dislocation density or disregistry vector. Here, $\rho_{i}^{(1)}, \rho_{i}^{(2)}$, and $\rho_{i}^{(3)}$ are the edge, vertical, and screw components of the general interplanar displacement density at the $i$ th nodal point, and $\gamma_{3}\left(\mathbf{f}_{i}\right)$ is the three-dimensional misfit potential. The corresponding components of the applied stress interacting with the $\rho_{i}^{(1)}, \rho_{i}^{(2)}$, and $\rho_{i}^{(3)}$, are $\tau^{(1)}=\sigma_{21}, \tau^{(2)}=\sigma_{22}$, and $\tau^{(3)}=\sigma_{23}$, respectively. $K, K_{e}$, and $K_{s}$ are the prelogarithmic energy factors defined earlier. The dislocation density at the $i$ th nodal point is $\rho_{i}=\left(f_{i}\right.$ $\left.-f_{i-1}\right) /\left(x_{i}-x_{i-1}\right)$, where $f_{i}$ and $x_{i}$ are the disregistry vector and the coordinate of the $i$ th nodal point (atomic row), respectively. The remaining quantities entering in this expression are $\chi_{i j}=\frac{3}{2} \phi_{i, i-1} \phi_{j, j-1}+\psi_{i-1, j-1}+\psi_{i, j}-\psi_{i, j-1}$ $-\psi_{j, i-1}$, with $\phi_{i, j}=x_{i}-x_{j}$, and $\psi_{i, j}=\frac{1}{2} \phi_{i, j}^{2} \ln \left|\phi_{i, j}\right|$. The quantity $L$ entering the last term is the outer cutoff radius for the elastic energy. ${ }^{16}$

The first term in the energy functional, $U_{\text {elastic }}$, represents the configuration-dependent (density or disregistry) part of the elastic energy, which has been discretized. Since any details of the displacements across the slip plane other than those on the atomic rows are disregarded, the dislocation density is constant between the nodal points. This explicit discretization of the elastic energy term removes the inconsistency in the original PN model and allows the totalenergy functional to be variational. Another modification in this approach is that the nonlinear misfit potential in the energy functional, $U_{\text {misfit }}$, is a function of all three components of the nodal displacements, $\mathbf{f}\left(x_{i}\right)$. Namely, in addition to the displacements along the Burgers vector, lateral and even vertical displacements across the slip plane are also included. This in turn allows the treatment of straight dislocations of arbitrary orientation in arbitrary glide planes. Furthermore, because the disregistry vector $\mathbf{f}\left(x_{i}\right)$ is allowed to change during the process of dislocation translation, the Peierls energy barrier can be significantly lowered compared to its corresponding value from a rigid translation. In order to examine the trend of energetics for different dislocations, we identify the dislocation configuration-dependent part of the total energy as the core energy, $U_{\text {core }}=U_{\text {elastic }}+U_{\text {misfit }}$, which includes the density-dependent part of the elastic energy and the entire misfit energy, in the absence of stress. The last term in Eq. (7), $K b^{2} \ln L$, is independent of the dislocation density, and hence it is irrelevant in the variational procedure. In fact, this term was not included in the total-energy expression of Ref. 15 because it does not contribute in the evaluation of the Peierls stress. The outer cutoff radius $L$ is $10^{3} \AA$ for all dislocations.

The response of a dislocation to an applied stress is achieved by minimization of the energy functional with respect to $\rho_{i}$ at the given value of the applied stress, $\tau_{i}^{(l)}$. An
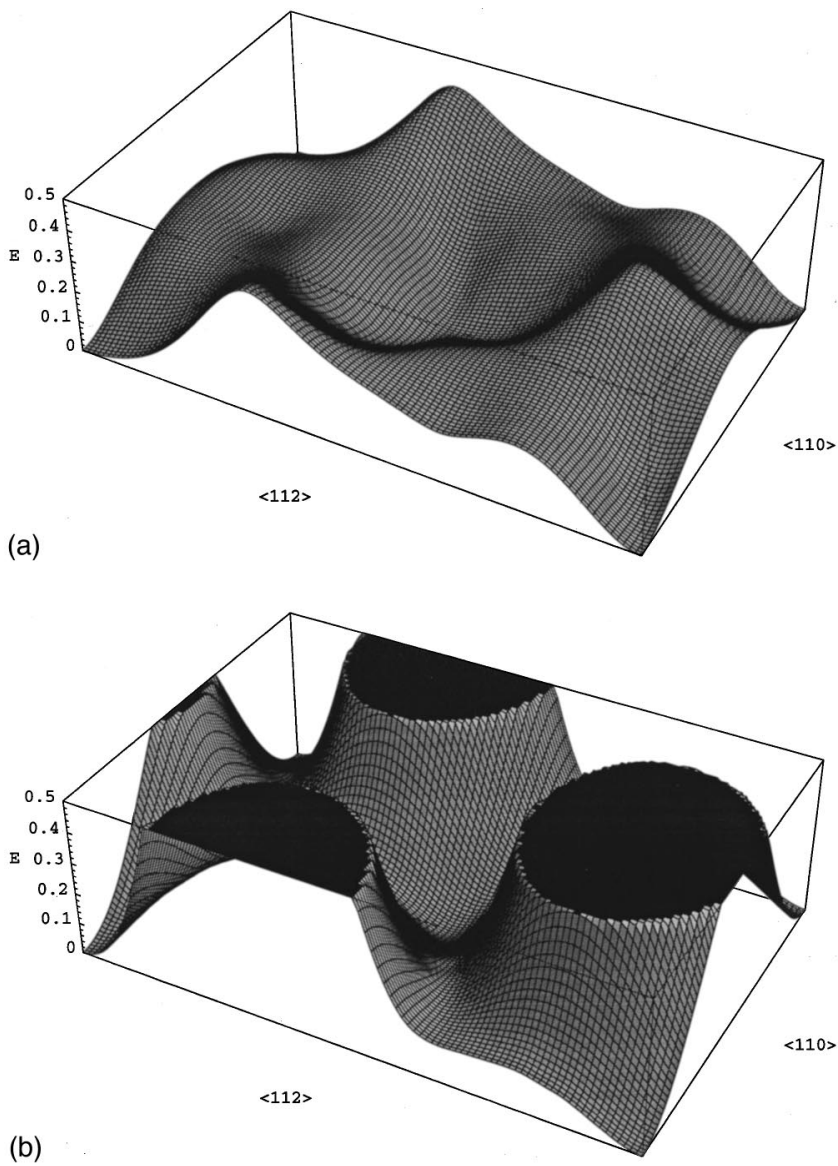

FIG. 2. The GSF energy surfaces for displacements along a (111) plane in $\mathrm{Al}\left(\mathrm{J} / \mathrm{m}^{2}\right)$ (the corners of the plane and its center correspond to identical equilibrium configurations, i.e., the ideal $\mathrm{Al}$ lattice): (a) from DFT pseudopotential plane-wave calculations; (b) from EAM calculations. The two energy surfaces are displayed in exactly the same perspective and on the same energy scale to facilitate comparison of important features. The EAM energy surface extends to values about four times larger than the maximum value displayed (black truncated regions).

instability is reached when an optimal solution for $\rho_{i}$ no longer exists, which is manifested numerically by the failure of the minimization procedure to convergence. The Peierls stress is defined as the critical value of the applied stress which gives rise to this instability.

\section{GENERALIZED-STACKING-FAULT ENERGY}

The first-principles GSF energy surface $\gamma(\mathbf{f})$ for the (111) plane was calculated on a grid of 40 points in the irreducible part of the (111) slip plane (1/12 of the area shown in Fig. 2). We used an augmented symmetrized polynomial basis to fit the calculated DFT GSF energy surface in order to facilitate the computation of dislocation properties. The basis was chosen so that it preserves the underlying translational and rotational symmetry of the fcc lattice. Because it is relatively faster to calculate the GSF energy using the EAM, ${ }^{24}$ we directly compute the GSF energy for any given disregistry vector.

Shown in Figs. 2(a) and 2(b) is the fitted GSF energy surface from the DFT and EAM, respectively. The three high peaks of the GSF surface correspond to the run-on stacking 

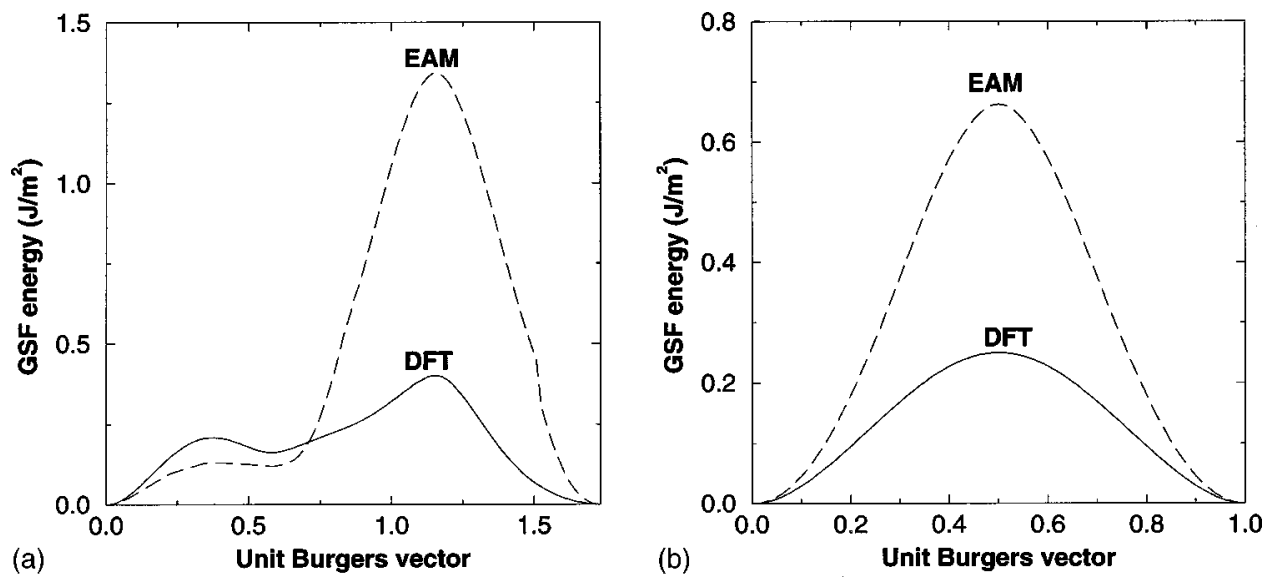

FIG. 3. Projections of the GSF energy surfaces on the (a) [12 $\overline{1}]$ and (b) [101] directions from both the DFT and EAM calculations.

fault configuration $A B C \mid C A B C$, in which two $C$ layers are neighboring to each other. The projection of the DFT and

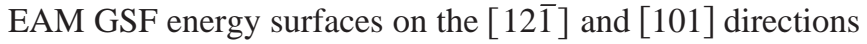
are shown in Figs. 3(a) and 3(b), respectively. The first en-

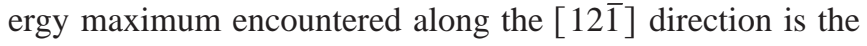
unstable stacking fault energy, which represents the lowest energy barrier for dislocation nucleation; ${ }^{25,26}$ the first energy minimum at $a_{0} / \sqrt{6}$ corresponds to the intrinsic stackingfault configuration, where a full dislocation dissociates into a pair of Shockley partials. The projection of the GSF surface on the [101] direction is symmetric with respect to the slip displacement of $a_{0} / 2 \sqrt{2}$. In both the DFT and EAM calculations, the unstable stacking-fault energy along [101] is found to be larger than that along [121] . This anisotropy of the unstable stacking-fault energy will affect the emission of dislocations from a crack tip. The difference between the DFT and EAM values for the run-on stacking fault is due to the fact that the $a b$ initio database to which the EAM potential was fitted does not include this fault configuration. The energy values of the various stacking faults obtained from the DFT and EAM calculations are summarized in Table I. The restoring stress for different directions using the DFT and EAM are shown in Figs. 4(a) and 4(b), respectively.

\section{DISLOCATION PROPERTIES}

In this section we present the results of various dislocation properties for the screw, $30^{\circ}, 60^{\circ}$ and edge dislocations with Burgers vector, $\mathbf{b}=a / 2[101]$, using the GSF energy surface obtained from the DFT and EAM calculations.

TABLE I. Fault vectors and energies for four stacking faults obtained from the DFT and EAM calculations. All energies are in $\mathrm{J} / \mathrm{m}^{2}$.

\begin{tabular}{lccc}
\hline \hline & Vector & DFT & EAM \\
\hline Intrinsic stacking & $1 / 6[12 \overline{1}]$ & 0.164 & 0.120 \\
Unstable stacking & $1 / 10[12 \overline{1}]$ & 0.224 & 0.141 \\
Unstable stacking & $1 / 4[101]$ & 0.250 & 0.663 \\
Run-on stacking & $1 / 3[12 \overline{1}]$ & 0.400 & 1.354 \\
\hline \hline
\end{tabular}

\section{A. Disregistry vector and dislocation width}

In order to examine the trend of the disregistry vector, $\mathbf{f}$ $=f_{1} \hat{x}+f_{2} \hat{y}+f_{3} \hat{z}$, as a function of the angle $\theta$ between the dislocation line and the Burgers vector $\mathbf{b}$, one needs to determine the components of $\mathbf{f}$ parallel and perpendicular to $\mathbf{b}$, i.e., $f^{a}=f_{1} \sin \theta+f_{3} \cos \theta$ and $f^{b}=f_{1} \cos \theta-f_{3} \sin \theta$, respectively. The results for $f^{a}$ using the DFT and EAM GSF surfaces are presented in Fig. 5(a) for the screw and $60^{\circ}$ dislocations, and in Fig. 5(b) for the $30^{\circ}$ and edge dislocations, respectively.

The dislocation half width $\zeta$, defined as the atomic distance over which $f^{a}$ changes from $\frac{1}{4} b$ to $\frac{3}{4} b$ (definition I), can be determined from Fig. 5. The half width may also be calculated from Eq. (6), which assumes a sinusoidal form for the restoring stress (definition II). While the first definition takes into account the full details of the entire GSF surface in the evaluation of the disregistry vector, the second definition involves only the maximum restoring stress $F_{\text {max }}$, which can be set equal to the first maximum of the restoring stress $F_{u s}$, encountered along the $[12 \overline{1}]$ direction. ${ }^{27}$ Values of $\zeta$ for the four dislocations evaluated from the above two definitions are listed in Table II. The half width increases monotonically with dislocation angle $\theta$ for both DFT and EAM calculations. The dislocation half width calculated from the EAM is larger compared to its corresponding DFT value, due to the smaller restoring force in the vicinity of both the unstable and intrinsic stacking faults [Fig. 4(a)]. Overall, and in particular for the DFT calculations, there is a good agreement for the values of $\zeta$ evaluated from the two definitions, which suggests that the details of the GSF surface are not important in the evaluation of $\zeta$. The larger discrepancy between the two definitions for the $60^{\circ}$ and edge dislocations using the EAM, is due to the fact that for both dislocations, the EAM energy surface gives dissociation into Shockley partials, which can only be manifested within the first definition.

\section{B. Dissociation behavior}

The dislocation density (solid curves) for the screw, $30^{\circ}$, $60^{\circ}$, and edge dislocations, calculated from the DFT GSF energy surface, are presented in Fig. 6, along with the corresponding results from the EAM calculations (dashed and dotted curves). In all cases, DFT predicts no splitting for the 

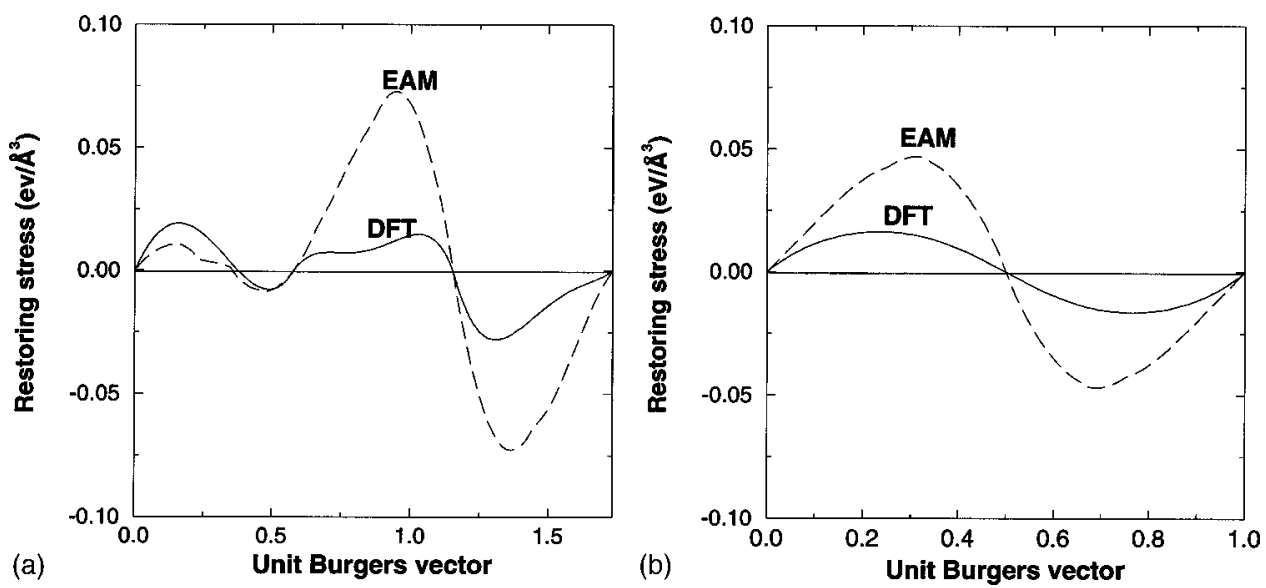

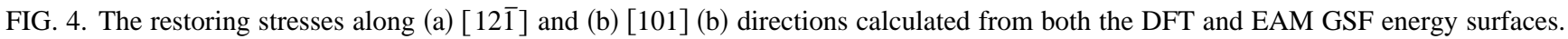

complete dislocations into partials, consistent with experiment. ${ }^{17}$ While the narrow double-peak structure found for the $60^{\circ}$ dislocation is suggestive of a splitting, it is rather due to the fact that the nodal points along the $X$ direction are not evenly spaced, i.e., they are distributed alternately by $\mathbf{b}_{p}$ and $\mathbf{b}_{p} / 2$, where $\mathbf{b}_{p}$ is the Burgers vector of the Shockley partial. This in turn gives rise to density fluctuations over the

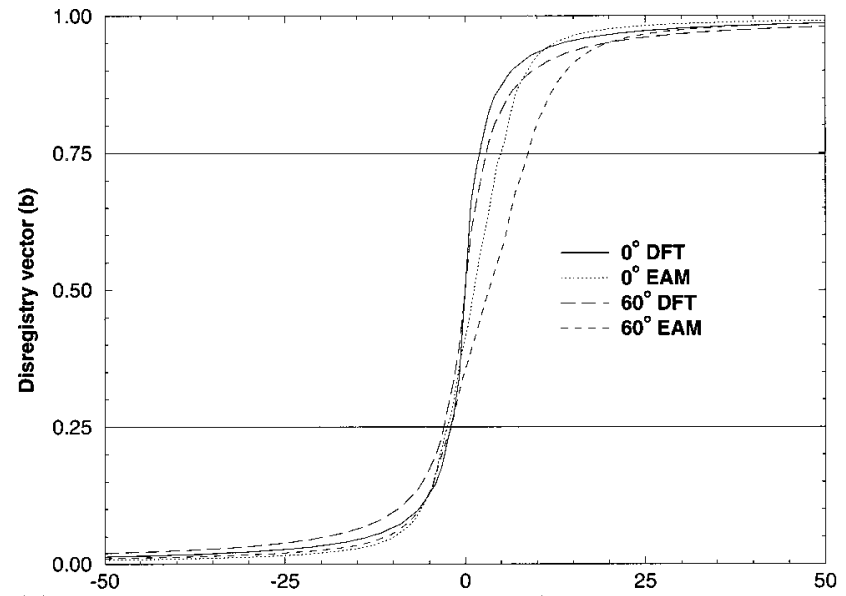

(a)

Nodal distance ( $(\AA)$

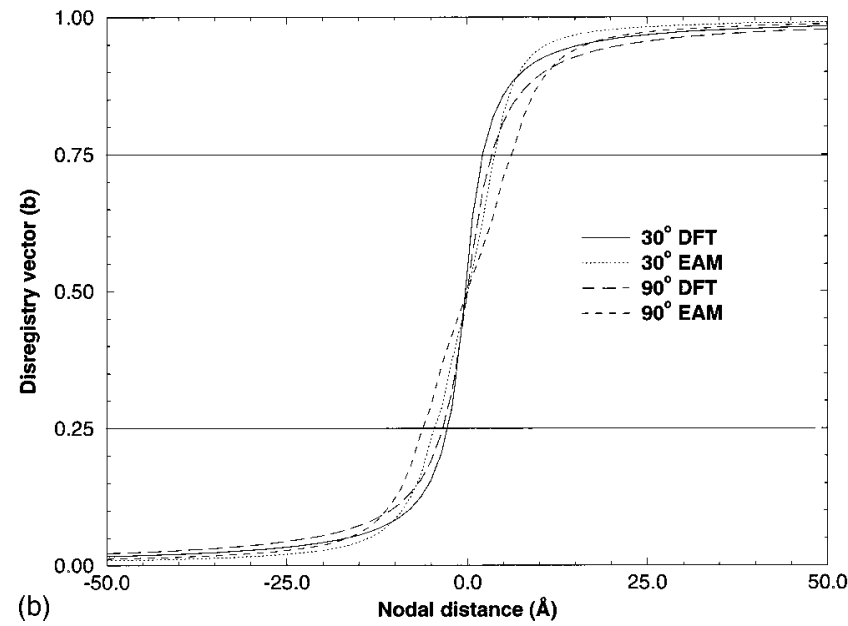

FIG. 5. The disregistry vector in units of the Burgers vector obtained from the DFT and EAM calculations for (a) the screw and $60^{\circ}$ dislocations; (b) the $30^{\circ}$ and edge dislocations. neighboring atomic rows and hence a double-peak structure. A double-peak structure may be indicative of a splitting, if the peaks are separated by a larger distance or the nodal points are evenly spaced, as in the case of the $30^{\circ}$ and edge dislocations.

In contrast to the DFT results and experiment, the EAM calculations predict that the full edge and $60^{\circ}$ dislocations dissociate into partials. In Fig. 6, we display both the screw and edge components for the $30^{\circ}$ and the $60^{\circ}$ dislocations. The multipeak found for the screw dislocation suggests that it might also be unstable. The $30^{\circ}$ dislocation exhibits a narrow double-peak structure. Overall, the dissociation trend found in the EAM calculations could be due to the smaller value of the intrinsic-stacking-fault energy. Recognizing that the EAM GSF energy surface differs from the DFT surface not only in the intrinsic stacking-fault (ISP) energy, but also in the energy profile around it, we have investigated the effect of the shape of the GSF surface on the dissociation behavior for the full edge dislocation using the DFT GSF surface. We have fixed the ISF energy of the GSF surface to its DFT value of $0.164 \mathrm{~J} / \mathrm{m}^{2}$, but varied the DFT energy profile around the ISF to make it as flat as possible to be similar to the that of the EAM GSF surface (inset of Fig. 7). Using the modified GSF surface, we then calculated the dislocation density for the edge dislocation, and found a small dissociation into partials (double peak in the density distribution) with a separation of one Burgers vector (Fig. 7). Therefore, not only the ISF energy itself, but also the GSF energy profile around it plays an important role on the dissociation behavior.

It is interesting to examine the character of the resultant partials in Fig. 6. The complete edge dislocation dissociates into two symmetric $60^{\circ}$ partials, whereas the $60^{\circ}$ dislocation dissociates into a $30^{\circ}$ (left double-peak) and a $90^{\circ}$ (right double-peak) partial. The double-peak structure again stems from the inequivalent nodal spacing between neighboring atomic planes. The fact that the density of the screw component (dotted curve) vanishes at the point where the edge component (right double peak) reaches its maximum, indicates the pure edge character of the $90^{\circ}$ partial. The $30^{\circ}$ dislocation exhibits a weak tendency for dissociation into a screw and a $60^{\circ}$ partial, while the edge component for the $60^{\circ}$ partial is singly peaked. Our result for the dissociation of 
TABLE II. Core half widths $\zeta$ (in $\AA$ ), with $\zeta^{I}$ from the definition through the disregistry vector and $\zeta^{I I}$ from the definition through the restoring stress; core energies $U_{\text {core }}$ and separate contributions from the configuration-dependent elastic energy, $U_{\text {elastic }}$ and $U_{\text {misfit }} ; K b^{2} \ln L$ (in eV/A); and Peierls stress (in $\mathrm{meV} / \AA^{3}$ ), for the four dislocations from both the DFT and EAM calculations. The last two rows list the Peierls stress for the screw and $60^{\circ}$ dislocations from direct atomistic simulations (At. Sim.) using the same EAM potential, and from the expression of Joós and Duesbery (JD) using the EAM values for $\zeta$ from the two definitions.

\begin{tabular}{llcccc}
\hline \hline & & Screw & $30^{\circ}$ & $60^{\circ}$ & Edge \\
\hline Core widths $\left(\zeta^{I}, \zeta^{I I}\right)$ & DFT & $(2.1,2.1)$ & $(2.5,2.4)$ & $(3.0,2.9)$ & $(3.5,3.2)$ \\
& EAM & $(3.7,3.4)$ & $(4.3,3.8)$ & $(5.4,4.7)$ & $(6.4,5.2)$ \\
$U_{\text {core }}$ & DFT & -0.0834 & -0.1096 & -0.1678 & -0.1979 \\
$U_{\text {elastic }}$ & EAM & -0.0534 & -0.0813 & -0.1413 & -0.1730 \\
& DFT & -0.1828 & -0.2317 & -0.3199 & -0.3666 \\
$U_{\text {misfit }}$ & EAM & -0.1679 & -0.2086 & -0.2960 & -0.3445 \\
$K^{2} \ln L$ & DFT & 0.0938 & 0.1221 & 0.1521 & 0.1688 \\
Peierls stress & EAM & 0.1145 & 0.1273 & 0.1547 & 0.1716 \\
& & 1.6050 & 1.8123 & 2.233 & 2.446 \\
& DFT & 1.60 & 0.33 & 0.61 & 0.02 \\
& EAM & 0.55 & 0.21 & 0.28 & 0.15 \\
& At. Sim. & 0.51 & & 0.29 & \\
\hline \hline
\end{tabular}

the full $60^{\circ}$ dislocation into partials agrees with direct atomistic simulations ${ }^{28}$ using the same Ercolessi-Adams EAM potential. Although this agreement indicates the success of the semidiscrete variational PN model in predicting the finer core structure, the result itself is not consistent with the experiment due to the smaller ISF energy from the EAM calculations. It is also interesting to compare our results to recent atomistic simulations of Mills et al. ${ }^{29}$ Employing the same Ercolessi-Adams EAM potential, Mills et al. determined the core spreading of the $60^{\circ}$ dislocation which in turn gives an ISF energy of $0.120 \mathrm{~J} / \mathrm{m}^{2}$, in excellent agreement with our EAM value (Table I). Furthermore, these authors conclude that empirical EAM potentials are not capable of accurately modeling the dissociation of the $60^{\circ}$ dislocation.

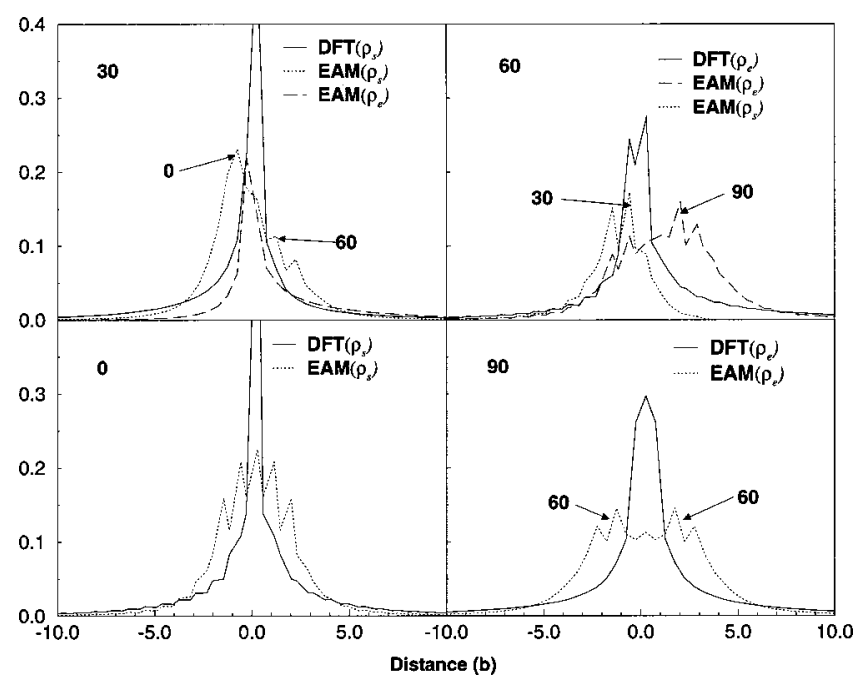

FIG. 6. Dislocation density for four dislocations (clockwise): screw, $30^{\circ}, 60^{\circ}$ and edge, obtained by using DFT and EAM calculations: For the $30^{\circ}, 60^{\circ}$, and edge dislocations, the characters of the resultant partials for the EAM calculations are indicated (see also text).

\section{Energetics and lattice resistance}

The DFT and EAM results for the energetics and the Peierls stress for the four dislocations are presented in Table II. The DFT results for the energetics are in good agreement with those from the EAM calculations, including the trend across the series. The misfit energy, $U_{\text {misfit }}$, increases monotonically from the screw to the edge dislocation, while the configuration-dependent elastic energy, $U_{\text {elastic }}$ (negative in sign) decreases as the angle increases. The configurationindependent elastic energy $K b^{2} \ln L$ is listed also in Table II. Several points need to be emphasized: (1) The configuration-

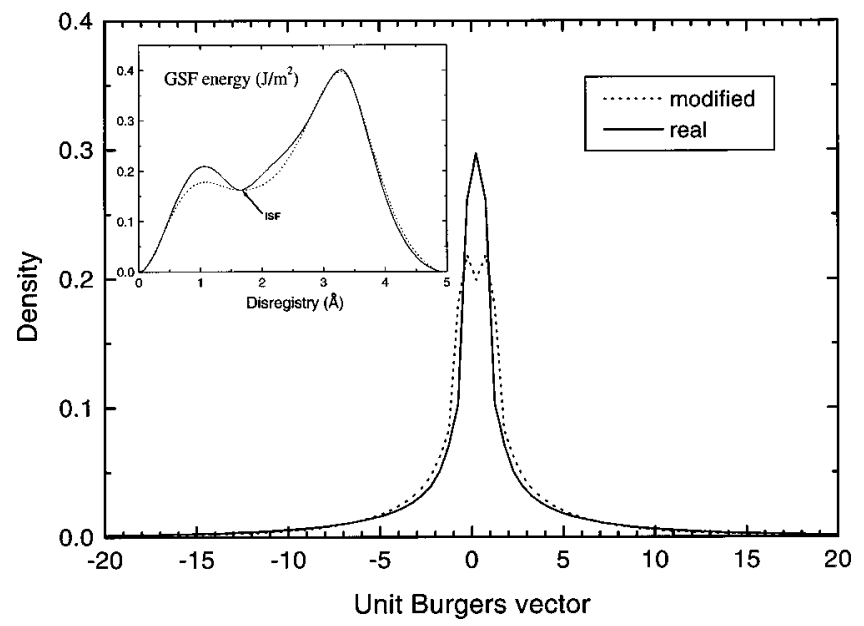

FIG. 7. Dislocation density for the edge dislocation calculated from the modified GSF energy surface (dashed line) and the DFT GSF energy surface without modification (solid line). The distance is in terms of the full Burgers vector. (Inset) The projection of the GSF energy surfaces along [12ī] with and without modifying the DFT energy profile. The solid curve represents the DFT energy profile while the dashed curve represents the modified GSF energy surface but keeping the same intrinsic stacking-fault energy as its DFT value. 
dependent elastic energy $U_{\text {elastic }}$, ignored in some previous studies, is the dominant contribution to the core energy $U_{\text {core }}$ (about a factor of two larger than $U_{\text {misfit }}$ ). More importantly, it depends strongly on the dislocation character; (2) While $U_{\text {elastic }}$ is negative here, in principle, it can be of either sign. For example, $U_{\text {elastic }}$ was found to be positive in $\mathrm{Si}^{32}{ }^{32}$ (3) Inclusion of the configuration-independent elastic term, $K b^{2} \ln L$, yields positive values for both the total energy and the total elastic energy.

The mobility of dislocations is characterized by the Peierls stress and the Peierls energy, the former being defined usually as the maximum derivative of the latter with respect to dislocation translation. As alluded earlier, the Peierls stress in this work is the critical value of the applied stress $\tau$, at which the dislocation energy functional fails to be minimized with respect to $\rho_{i}$ through standard conjugate gradient techniques. This approach is more accurate and physically transparent, because it captures the nature of the Peierls stress as the stress at which the displacement field of the dislocation undergoes a discontinuous transition. Furthermore, this definition for the Peierls stress treats the full and partial dislocations on an equal footing.

The Peierls stress calculated from the DFT GSF energy surface is in good agreement with that from the EAM calculations, except for the edge dislocation, where the EAM result is an order of magnitude larger than the DFT result. This discrepancy could be associated with the splitting of the edge dislocation into partials. Benoit et al. proposed ${ }^{30}$ that if the equilibrium separation between the two Shockley partials is an integer or half integer multiple of the vector $(a / 2)\langle 110\rangle$, so that the two partials move in phase and reach the troughs or the crests of the Peierls potential simultaneously, then the Peierls stress required to move the extended configuration is simply the stress required to move an isolated partial dislocation. On the other hand, if the two partials are rigidly separated by $(1 / 4)$ or $(3 / 4)$ times the vector $(a / 2)\langle 110\rangle$, then the partials are exactly out of phase and the Peierls stress on each partial is always equal and opposite, and hence the applied stress required to move the rigid configuration vanishes.

We next investigate the effect of dissociation on the Peierls stress. Since the EAM potential gives rise to dissociation for the perfect edge dislocation into two symmetric $60^{\circ}$ partials with a separation of about three times the Burgers vector (see Sec. VB), one can infer from the work of Benoit et al. that the Peierls stress for each partial is equal to the Peierls stress required to move the extended dislocation, i.e., the two partials and the intrinsic stacking fault in between. Continuum elastic theory gives the partials separation as

$$
d=\frac{\mu b_{p}^{2}}{8 \pi \gamma_{i s f}} \frac{2+\nu}{1-\nu}
$$

where $b_{p}$ is the partial Burgers vector and $\gamma_{i s f}$ is the intrinsic stacking-fault energy. ${ }^{16}$ Since the DFT GSF energy surface predicts no dissociation for the edge dislocation, one may force the dissociation by either increasing the shear modulus $\mu$ or reducing the intrinsic stacking fault energy $\gamma_{i s f}$.

Increasing $\mu$ by up to a factor of ten, but keeping the same DFT GSF energy surface, results in a wider dislocation density distribution, but yields no dissociation. On the other hand, keeping the same shear modulus $\mu$, but reducing the DFT value of $\gamma_{i s f}$ from 0.164 to $0.096 \mathrm{~J} / \mathrm{m}^{2}$ (the vicinity of the ISF in the GSF surface is also reduced by this energy rescaling), results in the dissociation of the edge dislocation into two $60^{\circ}$ partials, separated by one Burgers vector. The corresponding Peierls stress is $0.33 \mathrm{meV} / \mathrm{A}^{3}$, an order of magnitude larger than the original value of $0.02 \mathrm{meV} / \AA^{3}$. Further reduction of $\gamma_{i s f}$ to $0.085 \mathrm{~J} / \mathrm{m}^{2}$, yields a larger partials separation of $3 b$, but the same Peierls stress of 0.33 $\mathrm{meV} / \AA^{3}$. Since our calculations give a partials separation which is an integer multiple of the Burgers vector, we can infer from Benoit et al. that the Peierls stress of $0.33 \mathrm{meV} / \AA^{3}$ should be equal to that for moving an isolated $60^{\circ}$ partial. Therefore, the origin for the discrepancy in Peierls stress between the DFT and EAM results is that the DFT calculations predict no dissociation for the edge dislocation and hence a very low Peierls stress, whereas the EAM calculations, due to the lower $\gamma_{i s f}$, predict a dissociation into partials and hence a much higher Peierls stress.

In Table II, we list also the values of the Peierls stress for the screw and $60^{\circ}$ dislocations obtained from direct atomistic simulations $^{28}$ employing the same EAM potential. A final corroboration of the success of the semidiscrete variational PN model is that, for both the screw and $60^{\circ}$ dislocations, the EAM GSF energy surface yields a Peierls stress in excellent agreement with that from direct atomistic simulation ${ }^{28}$ using the same EAM potential. Furthermore, listed in Table II are the values of $\sigma_{P}$ calculated from the expression of Joós and Duesbery $^{31}$

$$
\sigma_{p}=\frac{2 \pi K b}{a^{\prime}} \exp \left(-\frac{2 \pi \zeta}{a^{\prime}}\right)
$$

Here, $a^{\prime}=2.47 \AA$ (for both dislocations) is the atomic spacing perpendicular to the dislocation line. Using the EAM values for $\zeta$ (from both definitions), Eq. (12) gives a Peierls stress for the screw dislocation and for the $60^{\circ}$ dislocation, both several orders of magnitude smaller than the corresponding values found from the current model and the results from direct atomistic simulations. On the other hand, had we used the DFT values for $\zeta$, Eq. (12) gives a Peierls stress comparable to that from the model calculations. This is due to the fact that Eq. (12), based on the sinusoidal approximation for the restoring force, can not treat dissociated dislocations.

\section{CORRELATION BETWEEN DISLOCATION PROPERTIES AND DISLOCATION CHARACTER}

In an effort to correlate dislocation properties with the dislocation character, we have studied, using the DFT GSF surface, the dislocation properties of 19 different dislocations that have the same Burgers vector but different orientations. The angle $\theta$ between the dislocation line and the Burgers vector varies from $0^{\circ}$ to $90^{\circ}$.

In Fig. 8, we present the half width of the dislocation core as a function of the dislocation angle, using both definitions described in Sec. V A. The half width increases monotonically with dislocation angle. Overall, the agreement is surprisingly good (less than 10\%), considering the fact that the first definition takes into account the details of the entire 


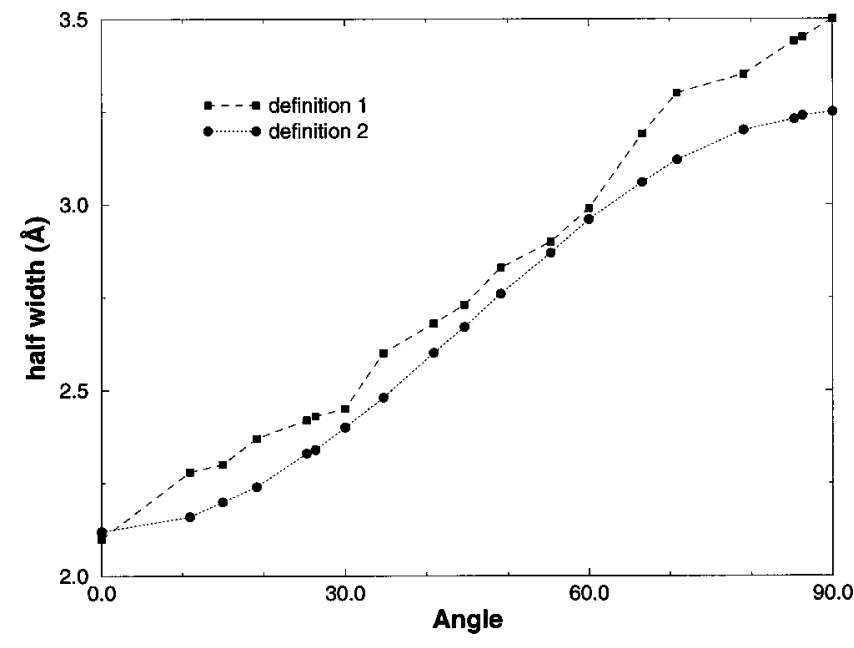

FIG. 8. The half width (in $\AA$ ) as a function of dislocation orientation using the two different definitions. The square stands for the definition through the disregistry vector and the dot stands for the definition through the restoring stress (only DFT results shown).

GSF surface, whereas the second definition employs only a single point (namely $F_{u s}$ ) of a simple sinusoidal GSF energy surface.

The core energy $U_{\text {core }}$, along with its separate contributions from the configuration-dependent elastic energy $U_{\text {elastic }}$ and the misfit energy $U_{\text {misfit }}$, are presented in Fig. 9 as a function of the dislocation angle $\theta$. We find that $U_{\text {core }}$ and $U_{\text {elastic }}$ decrease monotonically as the angle increases, whereas $U_{\text {misfit }}$ increases with $\theta$. The configurationdependent elastic energy $U_{\text {elastic }}$ decreases with $\theta$ because the prelogarithmic factor $K$ increases with $\theta$. On the other hand, the monotonic increase of $U_{\text {misfit }}$ with $\theta$ is due to the fact that the core width increases with the dislocation angle. Note, that the configuration-dependent elastic energy, not only is the dominant contribution to the total energy stored in the core region, but also is more sensitive to the dislocation character than the misfit energy.

In order to correlate the Peierls stress with the dislocation character, plotted in Fig. 10 is $\ln \left(\sigma_{p} \bar{a} / K b\right)$ as a function of $\zeta / \bar{a}$. Here, $\zeta$ is the half width of the dislocation core calculated from definition I and $\bar{a}$ is the average nodal spacing

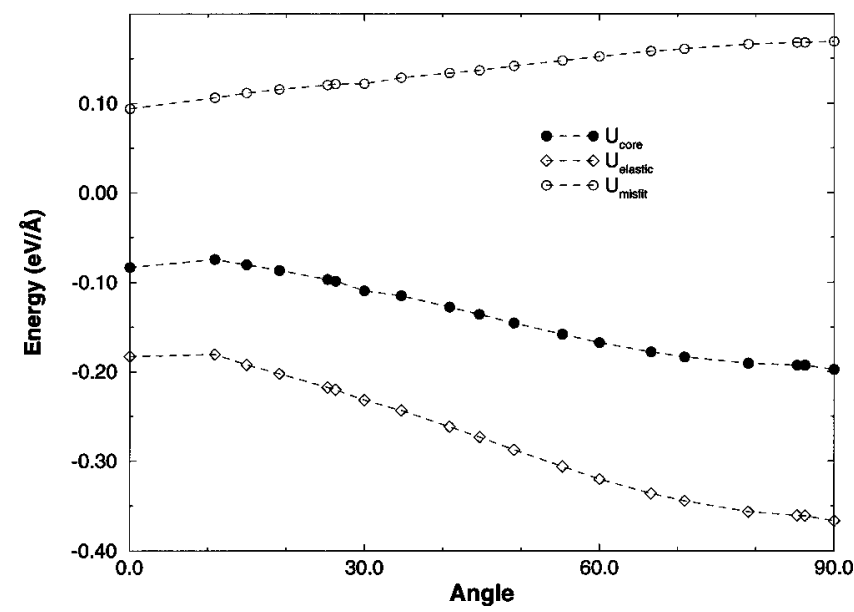

FIG. 9. The core energy, elastic energy, and misfit energy as a function of dislocation orientations.

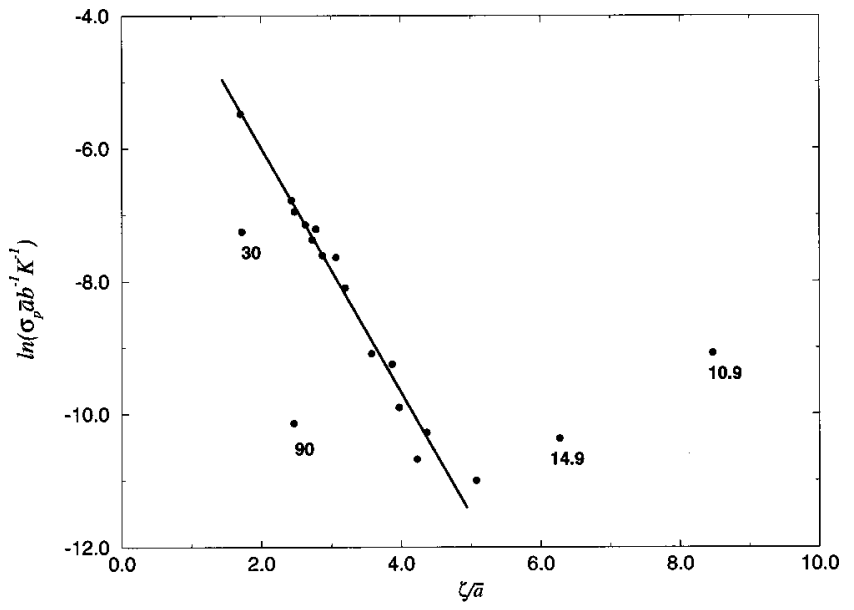

FIG. 10. The scaled Peierls stress as a function of the ratio of the core width to the average atomic spacing perpendicular to the dislocation line.

along the $X$ direction. It should be pointed out that most of the dislocations in the fcc lattice have noneven nodal spacings, except for the $30^{\circ}$ and edge dislocations. Most of the calculated values can be fitted (solid line) with

$$
\sigma_{p}=\frac{2 \pi K b}{\bar{a}} e^{-1.7 \zeta / \bar{a}}
$$

The large deviation of $\sigma_{P}$ for the $30^{\circ}$ and edge dislocations from the common trend, indicates that the nodal spacing (even versus non-even) between atomic planes plays an important role on the Peierls stress. Recently, we have developed $^{32}$ a general formalism which takes into account the effect of noneven nodal spacing in alternating lattices, such as the fcc lattice, and have shown that the Peierls stress for evenly spaced dislocations is several orders of magnitude lower than that for nonevenly spaced dislocations. On the other hand, the deviation of the $10.9^{\circ}$ and $14.9^{\circ}$ dislocations from the common trend is unclear at present. Note, that the Peierls stress is more sensitive to the average atomic spacing $\bar{a}$ than to the half width. For example, while both the $0^{\circ}$ and $14.9^{\circ}$ dislocations have predominant screw components and similar half widths of 2.1 and $2.3 \AA$, respectively, they have quite different atomic spacings, 1.2 and $0.3 \AA$, respectively. This results in a Peierls stress of $0.04 \mathrm{meV} / \AA^{3}$ for the $14.9^{\circ}$ dislocation, almost two orders of magnitude smaller than that of $1.60 \mathrm{meV} / \AA^{3}$ for the screw dislocation.

In conclusion, we have performed DFT and EAM calculations to obtain the GSF energy surfaces for the (111) glide plane of Al. From those calculations we extracted the core properties for various dislocations, using the semidiscrete variational generalization of the PN model. We have demonstrated that although the EAM gives the general trend for various dislocation properties, it fails to predict the correct finer structure of the dislocation core, i.e., the presence or absence of dissociation into partials, which in turn determines the mobility of dislocations. Since the dislocation dissociation into partials depends strongly on the intrinsic stacking-fault energy, direct atomistic simulations based on empirical potentials may also fail to predict the correct dis- 
sociation behavior. Thus, the results of the present work indicate that accurate DFT calculations of the GSF surface combined with the semidiscrete variational PN model enable the study of dislocation core properties more accurately and expediently. Moreover, this model can be extended to study a wide range of problems that are associated with more complex dislocations processes such as cross slip, dislocation intersections, reactions, etc.

\section{ACKNOWLEDGMENTS}

The work at California State University Northridge was supported by Grant No. DAAG55-97-1-0093 through the U.S. Army Research Office. The work of E.K. was supported by the Harvard Materials Research Science and Engineering Center, which was funded by NSF Grant No. DMR-9400396.
${ }^{1}$ M. S. Duesbery and G. Y. Richardson, CRC Crit. Rev. Solid State Mater. Sci. 17, 1 (1991).

${ }^{2}$ V. Vitek, Prog. Mater. Sci. 36, 1 (1992).

${ }^{3}$ V. Bulatov, F. F. Abraham, L. Kubin, B. Devincre, and S. Yip, Nature (London) 391, 669 (1998).

${ }^{4}$ B. Joós, Q. Ren, and M. S. Duesbery, Phys. Rev. B 50, 5890 (1994)

${ }^{5}$ Y. Juan and E. Kaxiras, Philos. Mag. A 74, 1367 (1996).

${ }^{6}$ J. Hartford, B. von Sydow, G. Wahnström, and B. I. Lundqvist, Phys. Rev. B 58, 2487 (1998).

${ }^{7}$ G. Schoeck, Philos. Mag. A 69, 1085 (1994).

${ }^{8}$ B. von Sydow, J. Hartford, and G. Wahnström, Comput. Mater. Sci. 15, 367 (1999).

${ }^{9}$ R. Peierls, Proc. Phys. Soc. London 52, 34 (1940).

${ }^{10}$ F. R. N. Nabarro, Proc. Phys. Soc. London 59, 256 (1947).

${ }^{11}$ J. D. Eshelby, Philos. Mag. 40, 903 (1949).

${ }^{12}$ V. Vitek, Philos. Mag. 18, 773 (1968).

${ }^{13}$ V. Vitek, Cryst. Lattice Defects 5, 1 (1974).

${ }^{14}$ R. Miller and R. Phillips, Philos. Mag. A 73, 803 (1996).

${ }^{15}$ V. V. Bulatov and E. Kaxiras, Phys. Rev. Lett. 78, 4221 (1997).

${ }^{16}$ J. P. Hirth and J. Lothe, Theory of Dislocations, 2nd ed. (Wiley, New York, 1992).

${ }^{17}$ M. S. Duesbery, Dislocations in Solids, edited by F. R. N. Na- barro (North-Holland, Amsterdam, 1989), Vol. 8, p. 67.

${ }^{18}$ P. Hohenberg and W. Kohn, Phys. Rev. 136, B864 (1964).

${ }^{19}$ W. Kohn and L. Sham, Phys. Rev. 140, A1133 (1965).

${ }^{20}$ J. Perdew and A. Zunger, Phys. Rev. B 23, 5048 (1984).

${ }^{21}$ H. J. Monkhorst and J. D. Pack, Phys. Rev. B 13, 5188 (1976).

${ }^{22}$ Y. Sun and E. Kaxiras, Philos. Mag. A 75, 1117 (1997).

${ }^{23}$ A. Wright, M. S. Daw, and C. Y. Fong, Philos. Mag. A 66, 387 (1992).

${ }^{24}$ F. Ercolessi and J. B. Adams, Europhys. Lett. 26, 583 (1994).

${ }^{25}$ J. R. Rice, J. Mech. Phys. Solids 40, 239 (1992).

${ }^{26}$ J. R. Rice and G. E. Beltz, J. Mech. Phys. Solids 42, 333 (1994).

${ }^{27}$ Due to the nonsinusoidal nature of the real GSF energy surface, no single value from the restoring stress surface can be justified to serve as $F_{\max }$. We arbitrarily chose $F_{u s}$ to be $F_{\max }$ by seeking the analogy between $F_{u s}$ and the unstable stacking-fault energy.

${ }^{28}$ V. V. Bulatov, O. Richmond, and M. V. Glazov, Acta Mater. 47, 3507 (1999).

${ }^{29}$ M. J. Mills, M. S. Daw, and S. M. Foiles, Ultramicroscopy 56, 79 (1994).

${ }^{30}$ W. Benoit, M. Bujard, and G. Gremaud, Phys. Status Solidi A 104, 427 (1987).

${ }^{31}$ B. Joós and M. S. Duesbery, Phys. Rev. Lett. 78, 266 (1997).

${ }^{32}$ G. Lu, N. Kioussis, V. Bulatov, and E. Kaxiras (unpublished). 